\title{
Arms pattern speed of galaxies in clusters
}

\author{
I. Rodrigues ${ }^{1}$, H. Dottori ${ }^{1}$ and D. Reichert \\ ${ }^{1}$ Instituto de Física, UFRGS,cp: 15051, cep: 91501-970, Porto Alegre, Brazil \\ email: dottori@if.ufrgs.br
}

\begin{abstract}
Disks of galaxies in clusters are deeply affected by interactions. We investigate the pattern speed of galaxies in cluster in order to detect a possible dependence with cluster environmental parameters, such as galaxies density. If the perturbation of cluster galaxies is mainly produced by the interaction with the cluster ambient, the pattern speed might well depend on the history of the galaxy orbit within the cluster. Tremaine \& Weinberg (1984) method is applied to 2-D $\mathrm{H}_{\alpha}$ velocity fields, reconstructed from the isovelocity contours published by Amram et al. (1992), and 2-MASS K-band images (Skrutskie 2001) to obtain spirals pattern angular speed. The use of $\mathrm{K}$-band images and $\mathrm{H}_{\alpha}$ velocity maps is justified by the fact that the perturbations imprinted in $\mathrm{H}_{\alpha}$ velocity maps are produced by the old stellar population which emits most of its energy in the near-IR. We analyzed Pegasus I cluster galaxies NGC 7593, NGC 7631 and NGC 7643 (this one shown in Figure 1). Preliminary results indicates that NGC 7593 presents a pattern speed $\Omega_{p} \sin (i)=18 \pm 5 \mathrm{~km} \mathrm{~s}^{-1} \mathrm{kpc}^{-1}$,(inclination $i=51^{\circ}$ ) while NGC 7631 and NGC 7643 pattern speeds are $\Omega_{p} \sin (i)=21 \pm 4 \mathrm{~km} \mathrm{~s}^{-1} \mathrm{kpc}^{-1}$ and $\Omega_{p} \sin (i)=35 \pm 3 \mathrm{~km} \mathrm{~s}^{-1} \mathrm{kpc}^{-1}$ (inclinations $i=64^{\circ}$ and $59^{\circ}$ respectively). In the three successful cases the correlation coefficients in the $\langle X\rangle$ vs $\langle V\rangle$ plots are $0.94,0.95$ and 0.98 respectively. We are presently analyzing other galaxies in the cluster.
\end{abstract}

Keywords. galaxies: spiral, galaxies: clusters
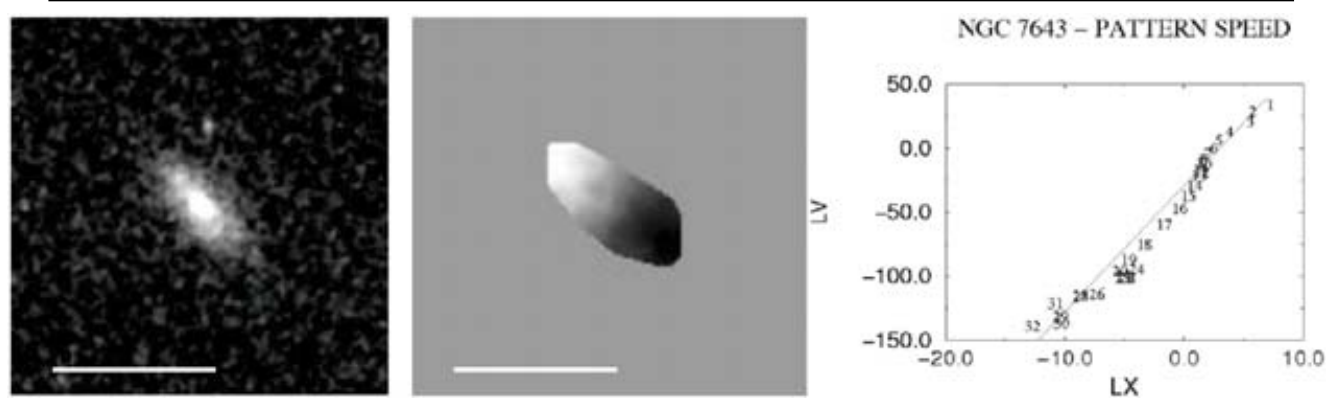

Figure 1. Left panel: 2MASS K-band image of NGC 7643. The white line is 1 arcminute long, corresponding to $16.6 \mathrm{kpc}$. Center panel: velocity map of NGC 7643. Right panel: plot $\langle X\rangle$ vs $\langle V\rangle$, from which $\Omega_{p}$ can be obtained.

\section{References}

Amram, P., Le Coarer, E., Marcelin, M., Balkowski, C., Sullivan, W. T., III, \& Cayatte, V. 1992, A\&AS 94, 175

Merrifield, M. R. \& Kuijken, K. 1995, MNRAS 274, 933

Skrutskie, M. F. 2001, BAAS 33, 827

Tremaine, S. \& Weinberg, M. D. 1984, ApJ 282, L5 